\title{
Exploring clustering of leprosy in the Comoros and Madagascar: A geospatial analysis
}

\author{
Nimer Ortuño-Gutiérrez ${ }^{\mathrm{a}, *}$, Aboubacar Mzembaba $^{\mathrm{b}}$, Stéphanie Ramboarinac, \\ Randrianantoandro Andriamira ${ }^{\mathrm{d}}$, Abdallah Baco $^{\mathrm{b}}$, Sofie Braet ${ }^{\mathrm{i}}$, Assoumani Younoussa $^{\mathrm{b}}$, \\ Bertrand Cauchoix ${ }^{\mathrm{c}}$, Zahara Salim ${ }^{\mathrm{b}}$, Mohamed Amidy ${ }^{\mathrm{b}}$, Saverio Grillone ${ }^{\mathrm{b}}$, \\ Tahinamandranto Rasamoelina ${ }^{\mathrm{e}}$, Emmanuelle Cambau ${ }^{\mathrm{f}, \mathrm{g}}$, Annemieke Geluk $^{\mathrm{h}}$, \\ Bouke C. de Jong ${ }^{i}$, Jan Hendrik Richardus ${ }^{j}$, Epco Hasker $^{i}$ \\ a Damien Foundation, Brussels, Belgium \\ ${ }^{\mathrm{b}}$ National Tuberculosis and Leprosy Control Program, Moroni, Comoros \\ ${ }^{\mathrm{c}}$ Raoul Follereau Foundation, Antananarivo, Madagascar \\ d National Leprosy Control Program, Antananarivo, Madagascar \\ e Centre d'Infectiologie Charles Mérieux, Université d'Antananarivo, Antananarivo, Madagascar \\ f INSERM, IAME UMR1137, Université de Paris, 75018 Paris, France \\ g APHP GHU Nord, Service de Mycocactériologie spécialisée et de référence, Centre National de Référence des Mycobactéries et de la Résistance des \\ Mycobactéries aux Antituberculeux - Laboratoire Associé, Paris, France \\ $\mathrm{h}^{\mathrm{h}}$ Leiden University Medical Center (LUMC), Department of Infectious Diseases, Leiden, The Netherlands \\ ${ }^{\mathrm{i}}$ Institute of Tropical Medicine, Antwerp, Belgium \\ ${ }^{\mathrm{j}}$ Department of Public Health, Erasmus MC, University Medical Center Rotterdam, Rotterdam, The Netherlands
}

\section{A R T I C L E I N F O}

\section{Article history:}

Received 19 March 2021

Received in revised form 30 April 2021

Accepted 7 May 2021

\section{Keywords:}

Clustering

Leprosy

Active case finding

Spatial analysis

\begin{abstract}
A B S T R A C T
Objectives: To identify patterns of spatial clustering of leprosy.

Design: We performed a baseline survey for a trial on post-exposure prophylaxis for leprosy in Comoros and Madagascar. We screened 64 villages, door-to-door, and recorded results of screening, demographic data and geographic coordinates. To identify clusters, we fitted a purely spatial Poisson model using Kulldorff's spatial scan statistic. We used a regular Poisson model to assess the risk of contracting leprosy at the individual level as a function of distance to the nearest known leprosy patient.

Results: We identified 455 leprosy patients; 200 (44.0\%) belonged to 2735 households included in a cluster. Thirty-eight percent of leprosy patients versus $10 \%$ of the total population live $\leq 25 \mathrm{~m}$ from another leprosy patient. Risk ratios for being diagnosed with leprosy were 7.3, 2.4, 1.8, 1.4 and 1.7, for those at the same household, at $1-<25 \mathrm{~m}, 25-<50 \mathrm{~m}, 50-<75 \mathrm{~m}$ and $75-<100 \mathrm{~m}$ as/from a leprosy patient, respectively, compared to those living at $\geq 100 \mathrm{~m}$.

Conclusions: We documented significant clustering of leprosy beyond household level, although $56 \%$ of cases were not part of a cluster. Control measures need to be extended beyond the household, and social networks should be further explored.

(c) 2021 The Author(s). Published by Elsevier Ltd on behalf of International Society for Infectious Diseases. This is an open access article under the CC BY-NC-ND license (http://creativecommons.org/licenses/by-nc-
\end{abstract}

$\mathrm{nd} / 4.0 /)$.

\section{Introduction}

Leprosy is an ancient infectious disease caused by Mycobacterium leprae, a microorganism discovered by Hansen (1874). The main transmission route is probably airborne (Araujo et al., 2016),

\footnotetext{
* Corresponding author at: Damien Foundation, Bd Leopold II, 263, B-1081 Brussels, Belgium.

E-mail address: Nimer.OrtunoGutierrez@damiaanactie.be (N. Ortuño-Gutiérrez).
}

with an incubation period ranging from 1-2 years to decades. When the disease is established, skin and nerve damage provoke the main symptoms. Immunological disturbances can trigger inflammatory episodes before, during and after treatment that can aggravate existing neuropathy or provoke new neuropathy ( $\mathrm{Wu}$ and Boggild, 2016). Delay in diagnosis and treatment of leprosy and its complications can result in permanent deformities (Yawalkar, 2009), which can cause social stigma (Grzybowski et al., 2016).

Multidrug therapy (MDT) with rifampicin, clofazimine and dapsone, introduced in the 1980s, has proven highly effective 
(World Health Organization, 2002; World Health Organization, 2019). As a result, leprosy prevalence has dramatically decreased worldwide and in 2000, leprosy was declared eliminated as a public health problem. Elimination was defined as a global prevalence of less than 1 leprosy case per 10,000 population (World Health Assembly, 1991). The impact on transmission, and by extension on the incidence of leprosy, has been less impressive. Since 2013, the annual number of new leprosy cases reported globally has persisted above 200000 , and the average incidence of leprosy in children has stagnated at close to 1 per 100000 inhabitants (World Health Organization, 2019). These 2 indicators support evidence of uninterrupted transmission of $M$. leprae.

The Global Leprosy Strategy 2016-2020 aimed to reduce transmission, focusing on early diagnosis, especially in children, and targeting endemic communities through active case finding strategies (World Health Organization, 2016). In 2018 the World Health Organization endorsed preventive treatment for close contacts of patients in the form of single-dose rifampicin postexposure prophylaxis (SDR-PEP) (World Health Organization, 2018). In Bangladesh, the pivotal COLEP trial had shown a $50 \%-$ $60 \%$ decrease in leprosy incidence when comparing contacts receiving SDR-PEP to contacts receiving a placebo (Ferreira et al., 2017; Moet et al., 2004b). More recently, a modelling study in the context of the Indian health system predicted that SDR-PEP is a cost-effective intervention based on its ability to prevent disabilities (Tiwari et al., 2020). Moreover, the feasibility of programmatic delivery of SDR-PEP has been demonstrated in 7 endemic countries in Asia, Africa and South America (Richardus et al., 2021).

The Post ExpOsure Prophylaxis for LEprosy (PEOPLE) trial that commenced in late 2018 aims to gather further evidence for SDRPEP and explore different modalities. The study is carried out in 64 villages in the Comoros and Madagascar, with 4 randomized study arms. Over 3 years ( 2 years in Madagascar), leprosy incidence in 3 intervention arms will be compared with that in the comparator arm without SDR-PEP (Arm 1). Modalities explored include provision of SDR-PEP to household contacts only (Arm 2), to all those living within $100 \mathrm{~m}$ of an index case (Arm 3), or to household contacts plus those living with $100 \mathrm{~m}$ of an index case and testing positive for antibodies directed against $M$. leprae-phenolic glycolipid-I (PGL-I), assessed by a rapid test that quantitatively detects immunoglobulin (IgM) antibodies against PGL-I in fingerstick blood (Arm 4) (Corstjens et al., 2019).

In this report, we analyse the baseline survey results, before SDR-PEP was provided, to assess patterns of spatial clustering of leprosy at household and individual level that may inform casefinding strategies.

\section{Methods}

\section{Study design}

As part of the baseline survey of the PEOPLE trial, we visited all households in 64 villages, 32 on Anjouan (Comoros), 16 on Mohéli (Comoros) and 16 in Miandrivazo district (Madagascar). All consenting household members were screened for leprosy and results were recorded, along with their leprosy history and basic demographic details, on smartphones, using a custom-designed app in Open Data Kit (ODK) Collect. The app also enabled the recording of the geographic coordinates of each household visited. Thus, we developed a dataset containing records of 102089 individuals, 57619 on Anjouan, 21982 on Mohéli and 22488 in Miandrivazo, divided over 20897 households. Median household size was 6 on Anjouan and Mohéli, with an interquartile range (IQR) of 4-8, and 5 in Miandrivazo (IQR 4-7). Among those surveyed, we found 455 (4.5 per 1000) prevalent leprosy cases, 346
(6.0 per 1000) on Anjouan, 39 (1.8 per 1000) on Mohéli, and 70 (3.1 per 1000) in Miandrivazo. We included cases already on treatment at the time of the survey and cases newly diagnosed during the survey. This dataset was used to explore the spatial clustering of leprosy.

\section{Study setting}

The Comoros is an archipelago located in the Indian Ocean, composed of 3 islands: the main island, Grand Comore, and two smaller islands, Anjouan and Mohéli. The country, with 832000 inhabitants, is 1 of 23 high-priority countries for leprosy identified by the World Health Organization (World Health Organization, 2019). The leprosy burden is concentrated on Anjouan and Mohéli, whereas Grand Comore has only sporadic cases. In 2018, the leprosy case notification rates were 6.6 and 7.4 per 10000 inhabitants, respectively, for Anjouan and Mohéli (Programme National de Lutte contre la Tuberculose et la Lèpre (PNTL), 2019). The Comoros is ranked 156 out of 189 countries on the human development index (UNDP, 2020). Over half of the population (55\%) depend on agriculture but soil erosion makes production insufficient and fishing partially fills the food gaps (The Independent Evaluation Office (IEO) of UNDP, 2019). In the latest national demographic survey, married women and men represent, respectively, $61 \%$ and $53 \%$ of the population that form nuclear families that include polygamic unions, which are more frequent in rural areas (20\% versus $14 \%$ in urban areas). (DGSP, 2014) Villages on the Comoros tend to be compact and well-delineated, with little open space inside village boundaries. Anjouan is the larger of the 2 leprosy endemic islands with a population of 332466 on a land area of $424 \mathrm{~km}^{2}$, versus 52360 population and a land area of 208 $\mathrm{km}^{2}$ for Mohéli. The population density on Anjouan is thus roughly 3 times higher than on Mohéli. This is further compounded by the fact that Anjouan has relatively little arable land because it is situated on a steep volcano. Most of the population therefore live in the coastal areas (Ratter et al., 2016).

Both passive and active case finding strategies for leprosy are implemented. For over 10 years, leprosy elimination campaigns have been conducted regularly. During campaigns, the population is invited for screening for skin conditions in a defined location. Presumptive leprosy patients are examined by specialized staff and contact screening is done in the same place (Ministère de la Santé, 2014). Throughout these campaigns, the proportion of new patients with visible deformities has been $<3 \%$ (World Health Organization, 2019) and the MDT completion rate for multibacillary cases (Programme National de Lutte contre la Tuberculose et la Lèpre (PNTL), 2019) has been $>90 \%$, indicative of high-quality leprosy services. Nevertheless, the continued high incidence of leprosy and the high proportion of children ( $<15$ years) among new cases $(>30 \%)$ illustrates uninterrupted transmission.

Madagascar is a much larger country located in the Indian Ocean, just south-east of the Comoros, with a land area of approximately $592,800 \mathrm{~km}^{2}$. It is also included among the $23 \mathrm{high}$ priority countries for leprosy, notifying approximately 1500 leprosy cases per year for a total population of 26.2 million. The leprosy burden is distributed unevenly with high and low prevalence areas. The proportion of new patients presenting with visible deformities is close to $20 \%$ and the MDT completion rate in multibacillary cases is $76 \%$; both indicators are explained by the limited geographical coverage of leprosy services. The proportion of children $<15$ years among new leprosy patients is $9 \%$ (World Health Organization, 2019), though lower than on the Comoros, it still illustrates persistent transmission. The Madagascar National Leprosy Control Program implements passive and active case detection. Active case detection is guided at the regional level by endemicity, geographical accessibility and available means. On the 
human development index Madagascar is ranked 164 out of 189 countries ((UNDP, 2020). At a national level, 63.4\% of the families are nuclear $(65.8 \%$ and $54.3 \%$ in the rural and the urban areas, respectively). The study district of Miandrivazo is a poor rural district with a land area of $12330 \mathrm{~km}^{2}$ and a population of 162462 . The study villages are situated $24-70 \mathrm{~km}$ from the district capital. They tend to be stretched out, consisting of multiple hamlets, and are often poorly accessible by road. Most villagers depend on agriculture for their livelihood, and raising livestock is another source of income (Institut National de la Statistique, 2020).

\section{Sample size and statistical analysis}

The sample size was calculated for the primary objective of the PEOPLE trial described earlier, which compares the effectiveness and cost-effectiveness of 3 different modalities of providing SDRPEP (Ortuno-Gutierrez et al., 2019b). A total of 64 villages were selected, with an estimated population of approximately 140000 . The actual numbers of inhabitants in the villages were lower than these estimates and, as mentioned earlier, 102089 participants were enrolled, among whom there were 455 active leprosy cases, either newly diagnosed during the survey or on treatment at that time.

Using the dataset described, we conducted 2 analyses. First, we used a collapsed dataset in which each household represented 1 observation with geographic coordinates, with the number of household members and leprosy cases as variables. This dataset was used to fit a purely spatial Poisson model using Kulldorff's spatial scan statistic to identify clusters of leprosy-affected households (Kulldorff and Nagarwalla, 1995). We used the software's default settings, i.e., circular clusters with a maximum size of $50 \%$ of the population. We retained only clusters that were statistically significant $(P<0.05)$ or borderline significant $(0.05 \leq P$ $\leq 0.10$ ).

For the second analysis, we used individual data. Making use of the distance matrix module in QGIS (Sutton et al., 2021), we determined for each individual the distance to the nearest other person who is a (prevalent) leprosy case. For individuals who were found to be leprosy cases themselves, this would be the distance to the nearest other leprosy case. Based on these distances, the study population was divided into 6 categories: 1 . Household contacts of a leprosy patient; 2 . Neighbours at $<25 \mathrm{~m}$ of a leprosy patient; 3 . Village contacts at $25-<50 \mathrm{~m}$ of a leprosy patient; 4 . Village contacts at $50-<75 \mathrm{~m}$ of a leprosy patient; 5 . Village contacts at $75-<100 \mathrm{~m}$ of a leprosy patient; 6 . Those living at $\geq 100 \mathrm{~m}$ of a leprosy patient. These data were used to fit a Poisson model with prevalent leprosy as the outcome and distance category as the predictor. As a reference category, we used those living at $\geq 100 \mathrm{~m}$ from the nearest known other person with leprosy. To account for intracluster correlation, we added village of residence nested within island location to the model as a random effect. As a sensitivity analysis, not foreseen in the original statistical analysis plan, we repeated the analysis by site (Anjouan, Mohéli and Madagascar) and also after excluding villages with zero prevalence.

\section{Results}

The 455 leprosy cases lived in 418 households. There was a single case in 382 households, 35 households had 2 cases, 1 household had 3 cases, and in the remaining 20479 households, no prevalent leprosy cases were found.

Prevalence rates by village were $0.0-30.8$ per 1000 with a median of 2.5 and IQR $0.99-5.0$ per 1000. In 8 out of 64 villages, no active cases were found. Figure 1 shows the distribution of leprosy prevalence by village during the baseline survey.
The Kulldorff's spatial scan statistic identified 4 statistically significant clusters on Anjouan, 1 significant and 1 borderline significant cluster on Mohéli, and 1 significant and 2 borderline significant clusters on Madagascar (Table 1). Of all 455 patients in the 3 sites, 200 (44.0\%) belonged to a high prevalence cluster though 15 of those patients were part of borderline statistically significant clusters. An example of a cluster in the Comoros is shown in Figure 2 below.

On Anjouan, 173/346 leprosy patients (50\%) were part of 4 high prevalence clusters, with statistically significant $P$-values of $<0.01$ and relative risks of 3.1-6.0. On Mohéli, 10/39 patients (24\%) belonged to 2 high prevalence clusters, 1 statistically significant cluster that includes $8 / 39$ cases (21\%) with a prevalence rate ratio of 42.4 and $P<0.0005$; the other 2 patients belong to a borderline significant cluster $(P=0.088)$ which is, in fact, a single household. In Madagascar, 17/70 patients (24\%) were part of 3 high prevalence clusters, 1 small but statistically significant cluster comprising 4 patients $(P=0.0045)$ and 2 marginally significant clusters made up of 7 and 6 patients with $P$-values of 0.067 and 0.072 , respectively. Details of the clusters are presented in Table 1.

In our second analysis, we found strong spatial clustering of leprosy based on the probability of being diagnosed with the disease as a function of geographical distance to the nearest other prevalent leprosy case at the time of the survey. In our model, controlling for intracluster correlation by using village of residence nested within island location as a random effect, and using those living at $\geq 100 \mathrm{~m}$ as a reference category, we observed prevalence rate ratios ranging from 7.5 for household members, 2.5 for neighbours within $25 \mathrm{~m}$, decreasing to 1.8 for those living at $75-100 \mathrm{~m}$, but all statistically significant. Details are shown in Table 2.

We repeated the analysis for each island individually and found similar associations and gradients, details are shown in Table 3. Due to the smaller sample size, the observed rates are less stable, but the general picture of associations and gradients observed remains unchanged. Excluding villages without cases had no significant impact either with prevalence rate ratios of 7.2 (95\% CI 5.0-10-3), 2.4 (95\% CI 1.7-3.4), 1.8 (95\% CI 1.3-2.5), 1.5 (95\% CI 1.0-2.1) and 1.7 (95\% CI 1.1-2.4), respectively, for household contacts, neighbours at $<25 \mathrm{~m}$ and neigbourhood contacts at $25-<50,50-<75$ and $75-<100 \mathrm{~m}$ distance, as compared with those living at $\geq 100 \mathrm{~m}$.

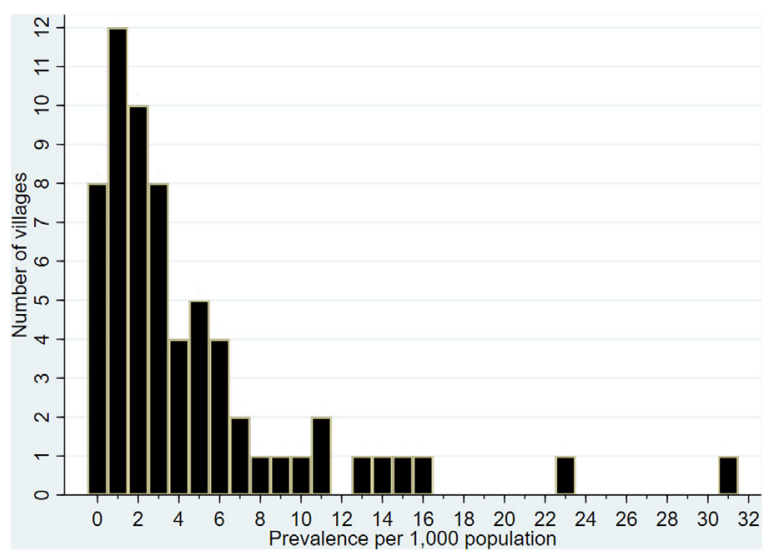

Figure 1. Distribution of leprosy prevalence per 1000 inhabitants, per village included in the survey, Comoros and Madagascar, 2019.

Among the 64 villages included in the study, there were 8 villages without leprosy cases. In the other 56 villages, the prevalence per 1000 population ranged between 1 in 12 villages to 31 in 1 village. 
Table 1

Characteristics of high prevalence clusters identified by Kulldorff's spatial scan statistic.

\begin{tabular}{|c|c|c|c|c|c|c|}
\hline Site & Cluster & Number of households & Cluster size (population) & Number of leprosy cases & Relative risk & $P$-value \\
\hline \multirow[t]{4}{*}{ Anjouan } & 1 & 355 & 1688 & 47 & 5.2 & $<0.0001$ \\
\hline & 2 & 11,59 & 6159 & 93 & 3.1 & $<0.0001$ \\
\hline & 3 & 91 & 464 & 16 & 6.0 & 0.0019 \\
\hline & 4 & 113 & 592 & 17 & 5.0 & 0.0078 \\
\hline \multirow[t]{2}{*}{ Mohéli } & 1 & 25 & 133 & 8 & 42.4 & $<0.0001$ \\
\hline & 2 & 1 & 4 & 2 & 297.0 & 0.088 \\
\hline \multirow[t]{3}{*}{ Madagascar } & 1 & 3 & 19 & 4 & 71.7 & 0.0045 \\
\hline & 2 & 45 & 236 & 7 & 10.5 & 0.067 \\
\hline & 3 & 32 & 160 & 6 & 13.1 & 0.072 \\
\hline
\end{tabular}

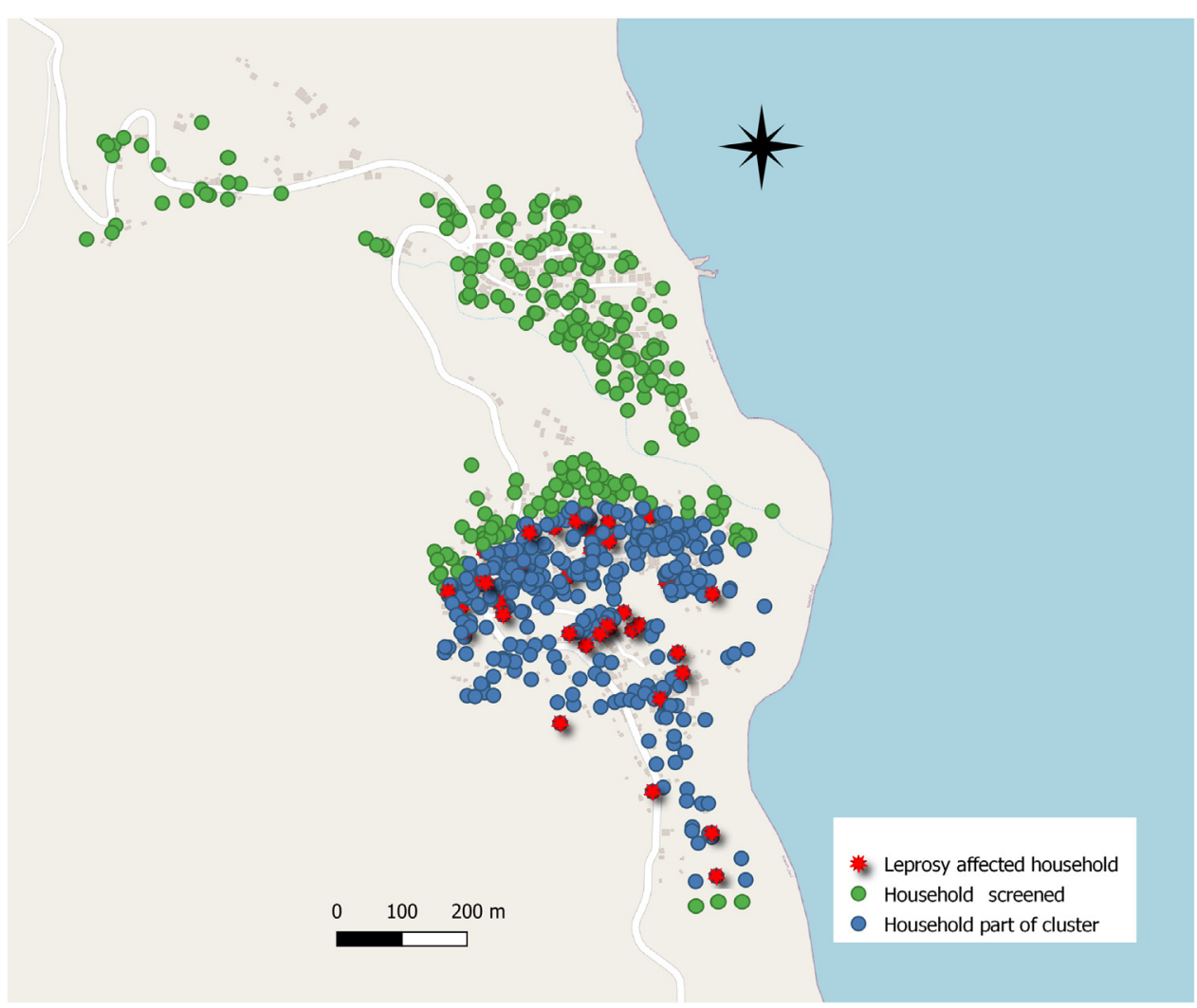

Figure 2. Clustering of leprosy cases in villages on Anjouan (with minimal adaptations to protect the privacy of participants).

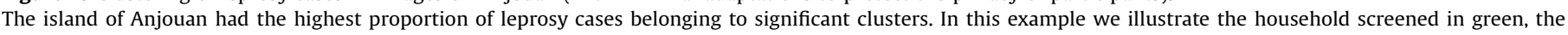
household screened and part of a cluster in blue and the leprosy-affected households in red.

\section{Discussion}

During our baseline survey in 64 villages on the Comoros and Madagascar, we found high leprosy prevalence and strong spatial clustering. The prevalence of leprosy at village-level exceeded 10 per 1000 population in 9 villages, including 1 in which $>30$ per 1000 had active leprosy. Among 455 leprosy patients, 200 (44\%) were part of high prevalence clusters. On Anjouan, where the prevalence is highest, these were most often large clusters extending across many households, even across villages. On Mohéli and in Madagascar clusters were smaller (2-8 patients). When considering the entire population in relation to the distance to the nearest index case, we found strong and highly significant associations. Compared with those living at $\geq 100 \mathrm{~m}$ from the

Table 2

Probability of being a leprosy patient as a function of distance to nearest index case random-effects model controlling for island and village of residence.

\begin{tabular}{|c|c|c|c|}
\hline Distance to index case & Population screened & Number of leprosy cases (\%) & Adjusted prevalence rate ratio $(95 \% \mathrm{CI})$ \\
\hline Same household & 2159 & $73(3.4)$ & $7.5(5.2-10.8)$ \\
\hline Neighbour contact at $<25 \mathrm{~m}$ & 9448 & $98(1.0)$ & $2.5(1.8-3.5)$ \\
\hline Neighbourhood contact at $25-<50 \mathrm{~m}$ & 13,645 & $91(0.7)$ & $1.9(1.4-2.7)$ \\
\hline Neighbourhood contact at $50-<75 \mathrm{~m}$ & 11,255 & $52(0.5)$ & $1.6(1.1-2.3)$ \\
\hline Neighbourhood contact at $75-<100 \mathrm{~m}$ & 8808 & $40(0.5)$ & $1.8(1.2-2.6)$ \\
\hline Neighbourhood contact at $\geq 100 \mathrm{~m}$ & 56,774 & $101(0.2)$ & Ref. \\
\hline
\end{tabular}


Table 3

Probability of being a leprosy patient as a function of distance to nearest index case random-effects model by island of residence, Anjouan, Mohéli, and Madagascar, 2019.

\begin{tabular}{lll}
\hline Distance to & \multicolumn{2}{l}{ Prevalence rate ratio $(95 \% \mathrm{CI})$} \\
\cline { 2 - 3 } Index case & Anjouan & Mohéli \\
\hline Same household & $6.4(4.0-10.1)$ & $32.8(12.6-85.3)$ \\
Neighbour at $<25 \mathrm{~m}$ & $2.4(1.5-3.7)$ & $5.4(1.9-15.8)$ \\
Neighbourhood contact at $25-<50 \mathrm{~m}$ & $2.0(1.3-3.1)$ & $2.0(0.6-6.9)$ \\
Neighbourhood contact at $50-<75 \mathrm{~m}$ & $1.8(1.1-2.8)$ & $2.1(1.0-4.5)$ \\
Neighbourhood contact at $75-<100 \mathrm{~m}$ & $1.6(1.0-2.7)$ & $2.5(0.8-7.5)$ \\
Neighbourhood contact at $\geq 100 \mathrm{~m}$ & Ref. & $0.6(0.2-1.8)$ \\
\hline
\end{tabular}

a There were no cases in this distance band on Mohéli.

nearest index case, the risk of leprosy was more than 7 times higher for household members. For neighbours at $<25 \mathrm{~m}$, the risk was 2-3 times higher. The association remained statistically significant up to $100 \mathrm{~m}$.

Our findings are consistent with earlier results from Anjouan in which we assessed the probability of being diagnosed with leprosy as a function of distance to index cases of earlier years, during a door-to-door survey conducted in 4 villages in 2017 (OrtunoGutierrez et al., 2019a,b). SDR-PEP had been provided in those villages in June 2015 to household contacts of leprosy patients diagnosed since the beginning of 2012. With those living at $>75 \mathrm{~m}$ from the nearest index case (diagnosed between January 2012 and June 2015) as a reference, the highest risk measured by incidence rate ratio (IRR) of current leprosy (in 2017) was found among those residing in the same household (IRR 2.4, 95\% CI 1.5-3.6) and a similar gradient with increasing distance was observed (IRR 1.8, 95\% CI 1.3-2.5, IRR 1.2, 95\% CI 0.8-1.7 and IRR 1.3, 95\% CI 0.8-2.1 for those living at 1-25, 26-50 and 51-75 m, respectively) (OrtunoGutierrez et al., 2019a,b). The fact that SDR-PEP had been provided to close contacts of index cases may have obscured part of the association, especially among household contacts. In an earlier study in Indonesia van Beers et al. (1999) reported an incidence rate ratio of 9.4 for household contacts. Moet et al. emphasise the importance of contact-related factors such as the closeness and intensity of the contact and inherited susceptibility when considering who to screen for leprosy (Moet et al., 2004a). Similar conclusions as those presented by our study were drawn by Moura et al., highlighting the importance of extending contact screening beyond the household (Moura et al., 2013).

Although we found significant spatial clustering of leprosy, it is important to realize that $56 \%$ of cases identified in our surveys were sporadic cases, not belonging to any cluster. We also found that of 455 patients identified, 284 (62.4\%) were living $>25 \mathrm{~m}$ from the nearest other leprosy patient and would have been missed if we had limited screening to near neighbours around an index case. Even a cut-off of at $100 \mathrm{~m}$ would still have missed $22 \%$ of our patients. In another high endemicity setting, in Bihar, India, a study also found significant clustering among household members and neighbours living up to $25 \mathrm{~m}$, with prevalence rate ratios of 6.3 (95\% CI 1.9-21) and 3.6 (95\% CI 1.3-10.2), respectively, when compared to those living at $>100 \mathrm{~m}$ (Hasker et al., 2019). Here too, the majority of leprosy cases (75\%) were at $>25 \mathrm{~m}$ from the nearest index case.

Geographic proximity is an easy-to-apply criterion for active case finding but will not be sufficient to interrupt transmission of $M$. leprae when a substantial proportion of patients are not part of any spatial cluster. We will have to consider other contacts than just near neighbours. Moet et al. also refer to the 'stone in the pond' principle applied in tuberculosis, which aligns with our findings and those of van Beers et al., who emphasise the importance of contact (van Beers et al., 1999; Moet et al., 2004a). Social network analysis and genotyping of $M$. leprae can play important roles in further elucidating transmission and identifying those most at risk. In a study in Brazil, $66 \%$ of leprosy cases belonged to a household social network with 3 or more leprosy cases (Boigny et al., 2020).

Our findings highlight the importance of redesigning active case finding strategies and targeting of post-exposure prophylaxis, taking into account the epidemiological burden and resources available. The tools used in this study (an app on a smartphone developed with open-access software) are well within reach of leprosy control programs. A shift away from paper-based systems could help to identify those most at risk more effectively.

Another area that should be explored is social network analysis. Although leprosy is a disease associated with social stigma, if all that is required from social contacts is to swallow one single dose of rifampicin to achieve a major reduction in risk, it is worthwhile trying to identify those contacts. In particular, this tracing would apply to contacts not living in the immediate surroundings of the index case.

As a limitation of our study, we acknowledge that despite the strong spatial correlations found, given the long incubation period of leprosy, it is highly likely that the source of the cases identified during our survey were patients that we did not consider as index cases because they were no longer on treatment when the survey took place.

Other neglected tropical diseases apply mass drug administration (MDA) for populations at high risk. However, this is harder to justify for a relatively rare disease like leprosy (Amoah et al., 2020). As time goes by, these MDA programs for once highly prevalent diseases are beginning to face similar challenges as in leprosy. If clusters of high endemicity could be clearly identified, strategies based on focused application of MDA could be considered. This was shown by Bakker et al. on small islands in Indonesia (Bakker et al., 2005) and is the strategy currently piloted in the third arm of the PEOPLE trial. The use of digital technology can be very helpful in outlining such clusters. Similar technologies have also been applied for monitoring and reporting coverage of MDA for other neglected tropical diseases (Oswald et al., 2020).

In conclusion, this study further supports the importance of expanding leprosy prevention and control activities beyond the household level. Focusing on those living within a 25 m perimeter of an index case is an efficient use of scarce resources but would miss a large proportion of cases. Additional criteria need to be developed and verified to identify those in need of screening and post-exposure prophylaxis, these could include social contacts. Digital tools can help in outlining high-risk areas, including in a programmatic context.

\section{Contributions}

NOG contributed to the conceptualization, formal analysis, writing the original draft and reviewing and editing integrating co- 
authors' contributions. AM, SR, RA, AB, SB, AY, BC, ZS, MA, SG, TR, $\mathrm{EC}, \mathrm{AG}$, and $\mathrm{BCJ}$ contributed to monitoring the study implementation and writing and reviewing the manuscript. $\mathrm{JH}$ and $\mathrm{EH}$ contributed to the conceptualization, supervision and validation of data analysis and visualization, and writing, reviewing and editing the manuscript.

\section{Funding source}

This study is part of the PEOPLE project, which is part of the EDCTP2 programme supported by the European Union (grant number RIA2017NIM-1847-PEOPLE). The views and opinions of authors expressed herein do not necessarily state or reflect those of EDCTP.

The study is also supported by a R2STOP Research grant from effect: hope, Canada and the Mission to End Leprosy, Ireland.

\section{Conflict of interest}

We declare no conflict of interest.

\section{Ethical approval}

This study is part of the PEOPLE trial, and it was conducted respecting the principles of the Declaration of Helsinki. The study has been approved by the Comité national d'Éthique pour les Sciences de la Vie et de la Santé in the Union of Comoros, and the Comité d'Éthique de la Recherche Biomédicale in Madagascar. It has also received the ethical approval of the Institutional Review Board of the Institute of Tropical Medicine as the sponsor of this study. In addition to these approvals, ethics approval was also received from the Ethics Committee of the University Hospital in Antwerp (Belgium). More detailed information about confidentiality is published in the data collection section of the PEOPLE protocol (Ortuno-Gutierrez et al., 2019a,b).

The PEOPLE trial is included in a public registry (7 September 2018, https://clinicaltrials.gov/ct2/show/NCT03662022).

\section{Acknowledgements}

We are grateful to the medical teams in the Comoros and Madagascar involved in the door-to-door active screening, particularly those belonging to the National Tuberculosis and Leprosy Programs of the Union of the Comoros and Madagascar, as well as to staff of Damien Foundation and Raoul Follereau Foundation.

\section{References}

Amoah AS, Hoekstra PT, Casacuberta-Partal M, Coffeng LE, Corstjens PLAM, Greco B, et al. Sensitive diagnostic tools and targeted drug administration strategies are needed to eliminate schistosomiasis. Lancet Infect Dis 2020;20(7):e165-72.

Araujo S, Freitas LO, Goulart LR, Goulart IM. Molecular evidence for the aerial route of infection of mycobacterium leprae and the role of asymptomatic carriers in the persistence of leprosy. Clin Infect Dis 2016;63(11):1412-20.

Bakker MI, Hatta M, Kwenang A, Van Benthem BHB, Van Beers SM, Klatser PR, et al. Prevention of leprosy using rifampicin as chemoprophylaxis. Am J Trop Med Hyg 2005;72(4):443-8.

Boigny RN, Souza Ead, Ferreira AF, Cruz JR, García GSM, Prado NmBdL, et al. Operational failures of leprosy control in household social networks with overlapping cases in endemic areas in Brazil. Epidemiol Serv Saúde 2020;29: e2019465.

Corstjens PLAM, van Hooij A, Tjon Kon Fat EM, Alam K, Vrolijk LB, Dlamini S, et al. Fingerstick test quantifying humoral and cellular biomarkers indicative for $\mathrm{M}$. leprae infection. Clin Biochem 2019;66:76-82.

DGSP II. Enquête démographique et de Santé et à indicateurs multiples aux Comores 2012. Available from:. 2014. https://dhsprogram.com/pubs/pdf/PR34/PR34.pdf.

Ferreira SMB, Yonekura T, Ignotti E, Oliveira LB, Takahashi J, Soares CB. Effectiveness of rifampicin chemoprophylaxis in preventing leprosy in patient contacts: a systematic review of quantitative and qualitative evidence. JBI Database Syst Rev Implement Rep 2017;15(10):2555-84.
Grzybowski A, Sak J, Pawlikowski J, Nita M. Leprosy: social implications from antiquity to the present. Clin Dermatol 2016;34(1):8-10.

Hansen GHA. Undersøgelser Angående Spedalskhedens Årsager (Investigations concerning the etiology of leprosy in Norwegian). Norsk Mag Laegervidenskaben $1874 ; 88$

Hasker E, Malaviya P, Scholar VK, de Koning P, Singh OP, Kansal S, et al. Post kala azar dermal leishmaniasis and leprosy prevalence and distribution in the Muzaffarpur health and demographic surveillance site. PLoS Negl Trop Dis 2019;13(10)e0007798.

Institut National de la Statistique. Recensement Général de la Population et de Habitation de Madagascar. 2020 Available from: https://www.instat.mg/ recensement-general-de-la-population-et-de-lhabitat-rgph/resultats-definitifs-rgph-3/. [Accessed 22 April 2020].

Kulldorff M, Nagarwalla N. Spatial disease clusters: detection and inference. Stat Med 1995;14(8):799-810.

Ministère de la Santé. Plan national de développement sanitaire 2015 - 2019 de l'Union des Comores. Comores: Ministère de la Santé de la Solidarité de la Cohésion Sociale et de la Promotion du Genre; 2014 p. 84.

Moet FJ, Meima A, Oskam L, Richardus JH. Risk factors for the development of clinical leprosy among contacts, and their relevance for targeted interventions. Lepr Rev 2004a;75(4):310-26.

Moet FJ, Oskam L, Faber R, Pahan D, Richardus JH. A study on transmission and a trial of chemoprophylaxis in contacts of leprosy patients: design, methodology and recruitment findings of COLEP. Lepr Rev 2004b;75(4):376-88.

Moura ML, Dupnik KM, Sampaio GA, Nobrega PF, Jeronimo AK, do NascimentoFilho JM, et al. Active surveillance of Hansen's Disease (leprosy): importance for case finding among extra-domiciliary contacts. PLoS Negl Trop Dis 2013;7 (3):e2093.

Ortuno-Gutierrez N, Baco A, Braet S, Younoussa A, Mzembaba A, Salim Z, et al Clustering of leprosy beyond the household level in a highly endemic setting on the Comoros, an observational study. BMC Infect Dis 2019a;19(1):501.

Ortuno-Gutierrez N, Younoussa A, Randrianantoandro A, Braet S, Cauchoix B, Ramboarina S, et al. Protocol, rationale and design of PEOPLE (Post ExpOsure Prophylaxis for LEprosy in the Comoros and Madagascar): a cluster randomized trial on effectiveness of different modalities of implementation of postexposure prophylaxis of leprosy contacts. BMC Infect Dis 2019b;19(1):1033.

Oswald WE, Kennedy DS, Farzana J, Kaliappan SP, Atindegla E, Houngbégnon P, et al. Development and application of an electronic treatment register: a system for enumerating populations and monitoring treatment during mass drug administration. Global Health Action 2020;13(1)1785146.

Programme National de Lutte contre la Tuberculose et la Lèpre (PNTL). Rapport annuel lèpre 2018. Moroni: Union des Comores; 2019 p. 2.

Ratter BMW, Petzold J, Sinane K. Considering the locals: coastal construction and destruction in times of climate change on Anjouan, Comoros. Nat Resour Forum 2016;40(3):112-26.

Richardus JH, Tiwari A, Barth-Jaeggi T, Arif MA, Banstola NL, Baskota R, et al. Leprosy post-exposure prophylaxis with single-dose rifampicin (LPEP): an international feasibility programme. Lancet Global Health 2021;9(1):e81-90.

Sutton T, Jacolin Y, Lanstorp J, Sherman GE, Duivenvoorde R, Athan T, et al. Quantum geographical information system (QGIS) user guide. 23.1.12. Vector analysis: 23.1.12.5. Distance matrix. 2021 Available from: https://docs.qgis.org/3.16/en/ docs/user_manual/processing_algs/qgis/vectoranalysis.html?highlight=matrix\#distance-matrix. [Accessed 27 February 2021].

The Independent Evaluation Office (IEO) of UNDP. Independent country programme evaluation: Union of the Comoros. 2019 Available from: https://erc.undp.org/ evaluation/documents/download/13763. [Accessed 22 April 2021].

Tiwari A, Blok DJ, Arif M, Richardus JH. Leprosy post-exposure prophylaxis in the Indian health system: a cost-effectiveness analysis. PLoS Negl Trop Dis 2020;14 (8)e0008521.

UNDP. The 2020 Human Development Report. The next frontier human development and the anthropocene. 2020 Available from: http://www.hdr.undp.org/ en/content/2020-human-development-report-next-frontier-human-development-and-anthropocene. [Accessed April 18 2021].

van Beers SM, Hatta M, Klatser PR. Patient contact is the major determinant in incident leprosy: implications for future control. Int J Leprosy Mycobact Dise 1999;67(2):119-28.

World Health Assembly. World Health Assembly 44. Resolutions and decisions. Leprosy. Available from:. 1991. http://www.who.int/neglected_diseases/ mediacentre/WHA_44.9_Eng.pdf?ua=1.

World Health Organization. Weekely epidemiological record. Global leprosy situation 2000. Geneva: Weekely Epidemiological Record; 2002 p. 8.

World Health Organization. Global leprosy strategy 2016 - 2020. Accelerating towards a leprosy-free world. 2016 Available from: https://apps.who.int/iris/ bitstream/handle/10665/208824/9789290225096_en.pdf?sequence=14\&isAllowed=y. [Accessed August 2019].

World Health Organization. Guidelines for the diagnosis, treatment and prevention of leprosy. 2018 Available from: http://apps.who.int/iris/bitstream/handle/ 10665/274127/9789290226383-eng.pdf?ua=1. [Accessed March 2020].

World Health Organization. Weekly epidemiological record. Global leprosy update, 2018: moving towards a leprosy-free world. 2019 Available from: https://www. who.int/wer/2019/wer9435_36/en/. [Accessed August 2019].

Wu J, Boggild AK. Clinical pearls: leprosy reactions. J Cutan Med Surg 2016;20 (5):484-5.

Yawalkar SJ. Deformities and their Management. In: Yawalkar SJ, editor. Leprosy for medical practitioners and paramedical workers. Basle, Switzerland: Novartis Foundation for Sustainable Development; 2009 p. 14. 